\title{
Compromiso académico estudiantil en tiempos de COVID-19: desafíos y oportunidades para la enseñanza en línea
}

\author{
Beatriz V. Tristán-Monrroy ${ }^{1}$, Isabel C. Flores-Rueda ${ }^{1}$, Armando Sánchez-Macías ${ }^{2 \star}$ y Guadalupe C. Briano-Turrent ${ }^{3}$ \\ (1) Coordinación Académica Región Altiplano, Universidad Autónoma de San Luis Potosí, México. \\ (correo-e: beatriz.tristan@uaslp.mx; isabel.flores@uaslp.mx) \\ (2) Coordinación Académica Región Altiplano Oeste, Universidad Autónoma de San Luis Potosí, México \\ (correo-e: armando.sanchez@uaslp.mx) \\ (3) Centro de Investigación y Estudios de Posgrado, Facultad de Contaduría y Administración, Universidad Autónoma de \\ San Luis Potosí, México (correo-e: guadalupe.briano@uaslp.mx) \\ * Autor a quien debe ser dirigida la correspondencia.
}

Recibido Jun. 2, 2021; Aceptado Jul. 26, 2021; Versión final Ago. 27, 2021, Publicado Dic. 2021

\begin{abstract}
Resumen
Los objetivos que se plantean en esta investigación son: a) evaluar los niveles de compromiso académico (CA) entre estudiantes de educación superior en modalidad en línea; b) validar el instrumento UWES-S en español (México) con estudiantes de educación superior; c) identificar si existen relaciones estadísticamente significativas entre las dimensiones de la variable y los aspectos sociodemográficos del estudiantado en educación en línea. La población de estudio es de 223 jóvenes potosinos mexicanos estudiantes de programas socio-administrativos. Los resultados muestran que existen dos tipologías de estudiantes: animados y desalentados. Las diferencias en CA están influenciadas por la institución de origen y se evidencia mayor CA conforme se avanza en la trayectoria académica. Existe una asociación entre orgullo por pertenecer a una carrera y la felicidad en las actividades inherentes a lo que se estudia. Se concluye que los resultados obtenidos permiten apoyar decisiones en la gestión escolar y pedagógica en procesos educativos mediados por las tecnologías de la información y la comunicación (TIC).
\end{abstract}

Palabras clave: compromiso académico; educación en línea; análisis factorial; tipologías; universitarios

\section{Student academic engagement in times of COVID-19: challenges and opportunities for online teaching}

\begin{abstract}
The main objectives of this research study are: a) to evaluate the levels of academic engagement (AE), in Spanish) in higher education students, b) to validate UWES-S for Spanish (Mexico) with higher education students, and c) to identify associations between AE dimensions and the socio-demographic characteristics of online students. The sample for the study is composed of 223 Mexican students from Potosi enrolled in social administration degrees. The results show that there are two types of students: encouraged and discouraged. Differences in AE levels are influenced by educational institution. There is evidence of increased $\mathrm{AE}$ as there is progress in academic trajectory. In addition, there is a correlation between pride in belonging to a degree and happiness inherent to the activities of the degree program. It is concluded that the results obtained serve to support decisions aimed at managing university and pedagogical education processes mediated by information and communication technologies (ICTs).
\end{abstract}

Keywords: academic engagement; online education; factorial analysis; typologies; university students 


\section{INTRODUCCIÓN}

El engagement académico o Compromiso Académico (CA) es un constructo que se manifiesta mediante una alta empatía y conexión hacia las actividades académicas, las cuales, se asumen en los estudiantes como un desafío positivo. Es lo contrario al agotamiento que genera el estrés académico (Mendes da Silva, et al., 2018). El CA, es un constructo que se conforma por tres factores: vigor, absorción y dedicación, se mide a través del cuestionario Utrecht Work Engagement Scale (UWES-S) de Schaufeli y Bakker (2004). Las propiedades psicométricas del engagement se han estudiado en diversas poblaciones objetivo en diversos países, principalmente en trabajadores y de manera emergente en estudiantes. En el caso de los primeros, se manifiesta mediante un sentido de conexión energética y de apego con su trabajo, con capacidades suficientes para afrontar las demandas de su actividad (Cachón-Zagalaz et al., 2018, Parra, et. al., 2020). Por su parte, en los estudiantes es una línea de investigación que está siendo abordada actualmente por su relevancia en los procesos educativos presenciales, pero también a distancia (Loscalzo y Giannini, 2019). Se interpreta como una actitud positiva que tiene la posibilidad de identificar las sensaciones experimentadas por los estudiantes con respecto a la educación que reciben. Un alto nivel de CA puede ser influyente para el bienestar académico en función de su trayectoria, además, se asocia con variables como rendimiento académico, satisfacción y absorción en los estudios, así como involucramiento (Hinrichs, et al., 2016).

La gestión del CA de estudiantes en educación en línea es compleja porque depende de factores como el diseño de las actividades de aprendizaje, pero también de la autorregulación del estudiante, ésta última fuera del control del docente (Barquerizo, et al., 2020). Sin embargo, existe evidencia de que la educación en línea permite mantener niveles adecuados de CA, permitiendo buenos resultados a través de herramientas didácticas como el aprendizaje cooperativo (Cotán-Fernández, et al., 2020). En la época en que la pandemia por el COVID-19 ha establecido condiciones en que una buena parte de los estudiantes han tenido que desarrollar sus labores académicas en línea o de manera virtual, las capacidades de autogestión y autonomía (autorregulación) cobran una gran relevancia. En este sentido, los niveles de CA son determinantes para, dadas las circunstancias, se logren resultados en el aprendizaje y desarrollo del alumnado de educación superior, los cuales, impactan en dimensiones que van más allá de la académica (Aspeé, et al., 2018). El contexto en que se desarrolla esta investigación es el de programas universitarios diseñados para ser operados bajo la modalidad presencial, pero que, debido a las restricciones que impuso la pandemia tuvieron que ser transferidos a una modalidad en línea. Esto implica clases sincrónicas a través de videollamada. Adicionalmente se utilizan otras herramientas de Tecnologías de la Información y la Comunicación (TIC) para compartir información y desarrollar actividades académicas. En estas universidades no existía un antecedente ni una planeación ante contingencias como la actual, por lo tanto, tampoco había un plan sistemático de formación en competencias digitales en profesores.

El engagement fue descrito por primera vez por Kahn (2008), nombrándolo engagement personal y describiéndolo como "[...] el aprovechamiento de los miembros de las organizaciones de sus propios roles de trabajo: en el engagement, las personas utilizan y se expresan a sí mismas física, cognitiva, emocional y mentalmente durante el desarrollo de sus roles" (p.700). Es también definido como "[...] un estado mental positivo y satisfactorio frente al trabajo, el cual se caracteriza por el vigor, dedicación y absorción del individuo" (Schaufeli, et al., 2002, p. 74), más bien persistente en el tiempo, pero caracterizado por que se vive de forma consciente en el presente. Para Schaufeli, et al. (2002) está integrado por tres componentes que se describen a continuación.

El factor de vigor hace referencia a niveles energéticos elevados y resistencia mental, resiliencia mientras se está en las horas de estudio, voluntad de esforzarse y persistencia, aun cuando se enfrenten a dificultades. Se refiere a la energía y se caracteriza por altos niveles de esta, así como resistencia y activación mental mientras se trabaja; el deseo y la predisposición de invertir esfuerzo en el trabajo que se está realizando y la persistencia incluso cuando aparecen dificultades en el camino (Schaufeli, et al., 2002; Schaufeli y Bakker, 2004). La dedicación implica estar altamente involucrado en el estudio, refiere a altos grados de implicación en el trabajo y con un alto sentido de significancia, así como un desafío para quien lo realiza. La dimensión dedicación revela la existencia de una alta implicación en la actividad que se realice, otorgándole un alto nivel de significado atribuido y un reto. Es decir, es involucrarse, entusiasmarse, estar orgulloso e inspirado en el trabajo, y se caracteriza por un sentimiento de importancia y desafío (Schaufeli, et al., 2002). Según Borzone (2017), en la medida que el estudiante se involucre de forma autónoma en su propio desarrollo académico podrá experimentar experiencias académicas satisfactorias, quienes administran su tiempo y estudian con dedicación obtienen mejores resultados.

Finalmente, la absorción se refiere a la concentración plena, inmersión y el disfrute de las actividades escolares, de forma que el estudiante no se percata del paso del tiempo y se deja llevar por las tareas académicas. La absorción ocurre cuando se está totalmente concentrado en la tarea, mientras se experimenta que el tiempo pasa volando y se tienen dificultades a la hora de desconectar de lo que se está haciendo, 
debido a las fuertes dosis de disfrute y concentración experimentadas. Por tanto, se caracteriza por un estado de concentración, de sentimiento de que el tiempo pasa rápidamente, así como dificultades para desligarse del trabajo debido a la sensación de disfrute y realización que se percibe (Schaufeli, et al. 2002; Schaufeli y Bakker, 2004).

\section{OTROS ANTECEDENTES}

En el contexto de la educación en línea, Martin y Bolliger (2018) han estudiado los niveles de CA en estudiantes de Estados Unidos de América (EUA), encontrando que las estrategias docente-estudiante son las que tienen mayor impacto en el CA, tales como la comunicación permanente mediante anuncios en la plataforma o por correo electrónico, así como proporcionar por anticipado las rúbricas para la evaluación. Afirman que los proyectos basados en casos reales, así como discusiones con guías estructuradas, son las estrategias que impactan en el CA de los estudiantes trabajando de forma independiente, destacando el papel de la autorregulación. Por otro lado, Dumford y Miller (2018) afirman que los estudiantes de EUA en educación en línea tienden a comprometerse menos en el aprendizaje colaborativo, interacción y discusión con sus pares, comparado a los estudiantes en clases presenciales; lo cual evidencia la importancia de las acciones que deben llevarse a cabo desde la gestión escolar. Cabe destacar que Diáz, et al. (2020) encontraron una asociación entre el CA y las tres dimensiones de los valores universales (autodirección, estimulación y benevolencia) en cadetes peruanos de dos escuelas militares, esto da cuenta de la implicación del CA más allá de los procesos educativos, sino que se relaciona con el contexto sociocultural del estudiante. Se puede hasta este punto destacar que el CA es un factor determinante para el éxito académico y que influye en procesos metacognitivos tales como la autorregulación, elemento clave -además de las estrategias pedagógico-didácticas- para lograr los resultados educativos cuando se implementa la educación en línea. También, que la educación en línea no es un reemplazo de la actividad presencial, sino que requiere de la formación y aplicación en principios didácticos, estrategias, actitudes y valores en el profesorado y estudiantado que deben ser desarrollados, bajo una planeación y acciones supervisadas y evaluadas.

En cuanto a la medición del CA, el instrumento Escala Utrecht de Engagement en el Trabajo (UWES, por sus siglas en inglés) es el más utilizado. Fue diseñado por Schaufeli et al. (2002) en base a una estructura trifactorial entre trabajadores y estudiantes, en los últimos, se utiliza una versión levemente refraseada para evaluar el CA. Según sus hallazgos, una estructura trifactorial presenta mejores indicadores de bondad de ajuste que una de uno o dos factores. En su investigación, reportan un índice de bondad de ajuste (GFI) de 0.91, un índice de ajuste comparativo (CFI) de 0.90 y un error de aproximación cuadrático medio (RMSEA) de 0.06. A partir de este punto, se identifica una convergencia en el uso y validación de este instrumento en sus diferentes versiones, en cuanto a número de ítems, como en sus adaptaciones a otros idiomas.

Wickramasinghe, et al. (2018) probaron la validez del UWES de 16 ítems en Sri Lanka en estudiantes, con una versión en idioma singalés traducida por un panel de expertos. Se encontró una consistencia interna adecuada y mediante el Análisis Factorial Exploratorio (AFE) se identificaron tres factores (vigor, dedicación y absorción) que explican el $65.4 \%$ de la varianza total. Kim, et al. (2017) utilizando el UWES-9 en una muestra de trabajadores coreanos, mostró que soluciones con las tres dimensiones: vigor, dedicación y absorción o agrupando en dos dimensiones (vigor y dedicación-absorción) fueron viables mediante el uso del AFE. Sin embargo, una vez realizado el Análisis Factorial Confirmatorio (AFC) se encontró un mejor ajuste en el modelo de tres factores, así como mejor consistencia interna y validez convergente y discriminante. La estructura factorial proporciona evidencia de validez asociada con el constructo, evaluando la coherencia entre el comportamiento teóricamente esperado y el comportamiento empírico.

Meng y Jin (2017) examinaron mediante el AFC la estructura factorial del UWES en estudiantes chinos, comprobando la confiabilidad de una estructura de tres factores y una consistencia interna de 0.91, afirmando con ello su utilidad y pertinencia para medir el CA. Por su parte, Loscalzo y Giannini (2019) en estudiantes italianos confirmaron la pertinencia psicométrica y validez del UWES-S-9 mediante el AFC, así como la posible relación entre las variables sociodemográficas y el desempeño académico con los niveles de CA. Reportan también evidencia concluyente de que estudiantes de áreas científicas y biomédicas muestran mayor dedicación hacia el estudio que aquellos del área de humanidades y ciencias de la salud. EI UWES-S-9 también ha sido validado mediante el uso del AFC con niveles de ajuste aceptables en un modelo unifactorial por Serrano, et al. (2019) con la versión en español ibérico en estudiantes de bachillerato. Además, identificaron que el sexo y la edad no impactan en el CA. Por otro lado, existe una asociación positiva entre el CA y el logro, la disposición y el bienestar; así como negativa con el estrés percibido.

La validez del UWES-S-J en su versión japonesa ha sido confirmada por Tayama, et al. (2019), con un modelo de un factor y de tres factores, siendo este último el que presentó un mejor ajuste, consistencia y confiabilidad en el test-retest en estudiantes. Reportan correlaciones positivas entre el resultado del UWES-S-J y el nivel de soporte social, resiliencia y felicidad subjetiva medidas con otras escalas. Portalanza, et al., (2017) 
validaron el UWES-9 en su versión en español en una muestra de estudiantes ecuatorianos. Mediante el método de estimación robusta, mostraron que un modelo de dos factores presenta mejor ajuste que uno de tres, así como su pertinencia para ser utilizado en el contexto Latinoamericano. Finalmente, otros hallazgos relevantes identificados mediante la aplicación del UWES son los de Díaz, et al., (2020), quienes en una base de datos en cinco países encontraron que la edad correlaciona con el CA de manera positiva siendo los de mayor edad los que presentan mayores niveles. En cuanto al sexo se reporta que los hombres muestran mayores niveles en la dimensión vigor, pero están parejos en las demás dimensiones.

EI UWES-Student (UWES-S) para medir CA en estudiantes universitarios ha experimentado un uso extendido, aunque poco se conoce en relación a sus propiedades psicométricas en el contexto mexicano y de la región, por lo tanto, pudiera mostrar variaciones debido al impacto sociocultural (Díaz, et al., 2020; Portalanza, et al., 2017). Así mismo, la educación en línea cuando no parte de un proceso de planeación tiene como potenciales factores de riesgo, la falta de habilitación en el profesorado (adecuadas estrategias pedagógico-didácticas) y estudiantado (habilidades de autorregulación), aunado a la falta de condiciones laborales apropiadas, infraestructura tecnológica, ambientes de aprendizaje y recursos para apoyar a los involucrados (Dumford y Miller, 2018; Martin y Bolliger, 2018). Estas condicionan el CA y a su vez, impiden el logro de resultados educativos satisfactorios (Cotán-Fernández, et al., 2020; Loscalzo y Giannini, 2019). Los objetivos que se plantean en esta investigación son: (a) evaluar los niveles de CA entre estudiantes de educación superior en modalidad en línea; (b) validar el instrumento UWES-S en su versión de español de México con estudiantes de educación superior e, (c) identificar si existen relaciones estadísticamente significativas entre las dimensiones de la variable y los aspectos sociodemográficos del estudiantado en educación en línea.

\section{METODOLOGÍA}

Los objetivos del presente estudio sobre evaluar los niveles de CA entre estudiantes de educación superior en una modalidad en línea que surge desde la adaptación por contingencia sanitaria, por ello es necesario validar el instrumento UWES-S, así como la identificación de relaciones entre dimensiones del CA y variables sociodemográficos del estudiante dirigen su atención a un diseño de investigación de enfoque cuantitativo con alcance descriptivo y causal de corte transversal. La obtención y recopilación de la información primaria para estudiantes universitarios potosinos se realizó a través de la aplicación personal de encuestas digitales en un ejercicio de retroalimentación en espacios de formación digitales de los estudiantes en colaboración con los profesores de distintas asignaturas de cada programa y como resultado se conformó una muestra de 223 estudiantes calculada a partir de información de la matrícula de estudiantes de los programas de administración pública, turismo sustentable e informática administrativa de la Universidad Intercultural (UI) y del programa de mercadotecnia de la Universidad Autónoma de San Luis Potosí (UASLP) que en total se estiman en 223 durante el mes de junio del 2020 -130 para el programa de mercadotecnia y 93 para los programas de Ul- considerando la fórmula estadística para poblaciones finitas con un nivel de confianza del $95 \%(p=q=0.5)$ y desviación estándar del 0.5 ,el tamaño de la muestra representativa es de 169 estudiantes; no obstante, se obtuvo una respuesta del $132 \%$ y se incluyeron 54 encuestas rectificando que no tuvieran errores de sesgo. Es importante detallar que las carreras en donde se aplicó el estudio no son planificadas para una modalidad digital, no obstante, por el contexto de la contingencia han tenido que ir ajustado los espacios de formación para responder a una demanda de enseñanza-aprendizaje en ambientes digitales.

La población fue de 223 jóvenes potosinos mexicanos estudiantes de programas socioadministrativos. En la descripción del perfil sociodemográfico se destacan; mujeres $(72.6 \%)$-hombres con $27.4 \%$ predominantemente entre los 17 a los 23 años (92.4\%) -24 años con 4\%, 25 años con $0.4 \%$, 26 años con 0.9\%, 28 años con $1.3 \%$ y 35 años con $0.9 \%$-. Las edades con mayor frecuencia son: 22 años (21.1\%), 18 años (17.5\%) y 20 años (15.7\%). Los estudiantes se encuentran inscritos en la UI (41.7\%) y la UASLP (58.3\%), ambas ubicadas en el altiplano del estado de San Luis Potosí, México. Los semestres que cursan los

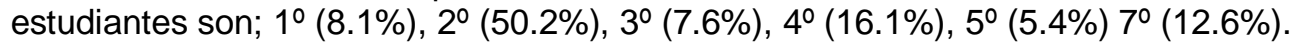

\section{Instrumento UWES-Student versión en español}

El instrumento utilizado es la versión del cuestionario UWES al contexto estudiantil en idioma español el cual es traducido desde la versión original holandesa se denomina UWES-Student (UWES-S) manteniendo la estructura de las dimensiones que el UWES (Parra, 2010). Puntualmente, para el presente estudio fue necesario buscar una versión de mayor adaptación al contexto mexicano la cual se encuentra en el estudio dirigido por Rodríguez y Oramas (2017) en Guanajuato para estudiantes de la licenciatura en ciencias de la actividad física y salud. La escala utilizada tiene 17 ítems divididos entre tres dimensiones: vigor (cinco ítems), dedicación (seis ítems) y absorción (seis ítems), las escalas de respuesta utilizada fueron de seis posiciones donde: $1=$ totalmente en desacuerdo, $2=$ en desacuerdo, $3=$ algo en desacuerdo, $4=$ algo de acuerdo, 5= de acuerdo y, $6=$ totalmente de acuerdo. 
Se asume la postura de Schaufeli y Bakker (2004), que conceptualiza al CA en tres dimensiones relevantes: vigor, dedicación, y absorción. El vigor (VI) se interpreta como niveles de energía altos y por tanto se demuestra con esfuerzo y persistencia constante en las asignaturas (Domínguez-Lara, et al, 2020), es decir, la voluntad de invertir esfuerzo y el enfoque de persistir a pesar de problemáticas (Carmona-Halty, et al., 2019). Mientras que la absorción (AB) se describe como la concentración en plenitud al realizar las actividades inherentes a la licenciatura (Domínguez-Lara, et al, 2020), así como definen Carmona-Halty, et al. (2019, p.1) "[...] absorto de lo que uno está estudiando, donde el tiempo pasa rápido". Finalmente, la dedicación (DE) se explica a partir del entusiasmo y el valor implicado a las actividades de la licenciatura (Domínguez-Lara, et al, 2020) además de estar involucrado en los estudios y experimentar importancia, orgullo y desafío de las actividades escolares (Carmona-Halty, et al., 2019). Es relevante mencionar que para el contexto universitario las dimensiones del CA tienen un sentido compuesto por elementos conductuales, cognitivos y emocionales (Portalanza, et al., 2017). En la tabla 1 se presenta la definición operacional de la variable y sus dimensiones.

Tabla 1. Dimensiones del CA (Fuente: Schaufeli y Bakker (2004)

\begin{tabular}{|c|l|c|}
\hline Códigos & Descripción & Dimensión \\
\hline AB1 & El tiempo "pasa volando" cuando realizo mis tareas como estudiante. & \multirow{2}{*}{ Absorción } \\
\hline AB2 & Olvido todo lo que pasa alrededor de mi cuando estoy abstraído con mis estudios. & \\
\hline AB3 & Soy feliz cuando estoy haciendo tareas relacionadas con mis estudios & \multirow{2}{*}{ Dedicación } \\
\hline AB4 & Es difícil para mí separarme de mis estudios & \\
\hline AB5 & Me "dejo llevar" cuando realizo mis tareas como estudiante. & \\
\hline DE1 & Creo que mi carrera tiene significado. & \\
\hline DE2 & Estoy entusiasmado con mi carrera. & \\
\hline DE3 & Mis estudios me inspiran cosas nuevas. & \multirow{2}{*}{ Vigor } \\
\hline DE4 & Estoy orgulloso de hacer esta carrera. & \\
\hline DE5 & Estoy inmerso en mis estudios. & \\
\hline DE6 & Mi carrera es retadora para mí. & \\
\hline VI1 & Mis tareas como estudiante me hacen sentir lleno de energía. & \\
\hline VI2 & Me siento fuerte y vigoroso cuando estoy estudiando o voy a clases. \\
\hline VI3 & Cuando me levanto por la mañana me apetece ir a clase o estudiar. \\
\hline VI4 & Puedo seguir estudiando durante largos periodos de tiempo. \\
\hline VI5 & Soy muy "resistente" para afrontar mis tareas como estudiante. \\
\hline VI6 & En mis tareas como estudiante no paro, incluso si no me encuentro bien. \\
\hline
\end{tabular}

Se realizaron análisis que permiten comprobar la validez de las escalas propuestas, un AFE y un AFC que como resultado obtuvieron una combinación de dos factores que permiten explicar el $66.82 \%$ de la varianza de la muestra. Además, se observa que los resultados de los análisis factoriales -tanto exploratorio como confirmatorio- pueden confirmar la validez y fiabilidad de la escala a través de los valores que arroja el índice alfa de Cronbach ( $\alpha$ ), la Fiabilidad Compuesta (FC), la Varianza Media Extractada (VME), los pesos y las cargas de las variables observadas en las variables latentes cuyo valor es superior a los 0.5 , lo cual es aceptable. En la tabla 2 se concentran los ítems y los atributos de las dimensiones que conforman al compromiso lo cual permite observar la consistencia interna a través de los índices $\alpha$, FC, y la VME, los pesos y las cargas de las variables observadas en las variables latentes.

Tabla 2. Medidas de validez y fiabilidad de la escala

\begin{tabular}{|c|c|c|c|c|c|c|c|}
\hline Factor y porcentaje de explicación acumulado & Código & Carga & Valort & $P$ & $\alpha$ & $F C$ & $V M E$ \\
\hline Dedicación & 1 & & & & \multirow{5}{*}{0.909} & \multirow{5}{*}{0.984} & \multirow{5}{*}{0.559} \\
\hline & 0.718 & 0.049 & 14.646 & *** & & & \\
\hline \multirow[t]{3}{*}{$31.07 \%$} & 0.87 & 0.054 & 16.235 & *** & & & \\
\hline & 0.995 & 0.048 & 20.929 & 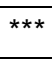 & & & \\
\hline & 0.716 & 0.053 & 13.421 & *** & & & \\
\hline \multirow[t]{2}{*}{ Vigor y absorción } & 1 & & & & \multirow{4}{*}{0.862} & \multirow{4}{*}{0.971} & \multirow{4}{*}{0.712} \\
\hline & 0.8 & 0.073 & 10.982 & $* * \star$ & & & \\
\hline \multirow[t]{2}{*}{$53.98 \%$} & 0.978 & 0.063 & 15.415 & 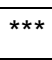 & & & \\
\hline & 0.789 & 0.064 & 12.314 & $\star * \star$ & & & \\
\hline
\end{tabular}


Posteriormente, se examinó la estructura factorial a través de un AFC mediante un enfoque de máxima verosimilitud, donde el modelo de dos factores de línea presentó el mejor ajuste aceptable. En detalle, se evaluó la bondad de ajuste mediante el cálculo de chi-cuadrado $\left(\mathrm{x}^{2} / \mathrm{gl}\right)$ con un resultado de 1.522 con $95 \%$ de intervalo de confiabilidad $\left(x^{2}=39.573, g l=26.0\right)$. La variación del $x^{2} / g l$ para la proporción aceptable se muestra altamente sensible a la muestra (Carmona-Halty, et al., 2019). Además, se tomaron en cuenta los valores el error de aproximación de la raíz al cuadrado medio (RMSEA) con un resultado de 0.048 el valor se considera aceptable de acuerdo con los criterios donde el valor de corto deberá ser menor a 0.07 así como el índice de ajuste comparativo (CFI) de 0.990 en donde el criterio se considera como buen ajuste (Hooper, et al., 2008). Para analizar la variable CA en la muestra se utilizaron las técnicas estadísticas de análisis de conglomerados k de medias y jerárquico, con el propósito de tipificar los grupos que se conforman dentro de la educación superior. También se plantearon pruebas de hipótesis para identificar relaciones estadísticamente significativas entre las dimensiones de la variable CA mediante el uso de la prueba coeficiente de contingencia de Pearson (C), U de Mann Whitney y H de Kruskall Wallis.

\section{RESULTADOS}

El inicio de la contingencia sanitaria provocó cambios drásticos en la dinámica de los espacios de formación, en consecuencias, estudiantes universitarios mexicanos dieron un giro brusco al aprendizaje remoto que se requirió de un día a otro, competencias digitales de aprendizaje en plataformas experimentales que tampoco estaban totalmente adecuadas para una experiencia así. Por lo anterior, fue necesario puntualizar en los resultados de los elementos de construyen el CA en estudiantes universitarios. Como se muestra en la figura 1 , en todos los casos las dimensiones del CA se encuentran por debajo en la UASLP con respecto a la UI, con diferencias que van del 13 hasta el 16\%. Los niveles de las tres dimensiones en la UASLP se consideran medios, mientras que en el caso de la Ul el vigor es medio, mientras que la $\mathrm{AB}$ y la $\mathrm{DE}$ son altos.

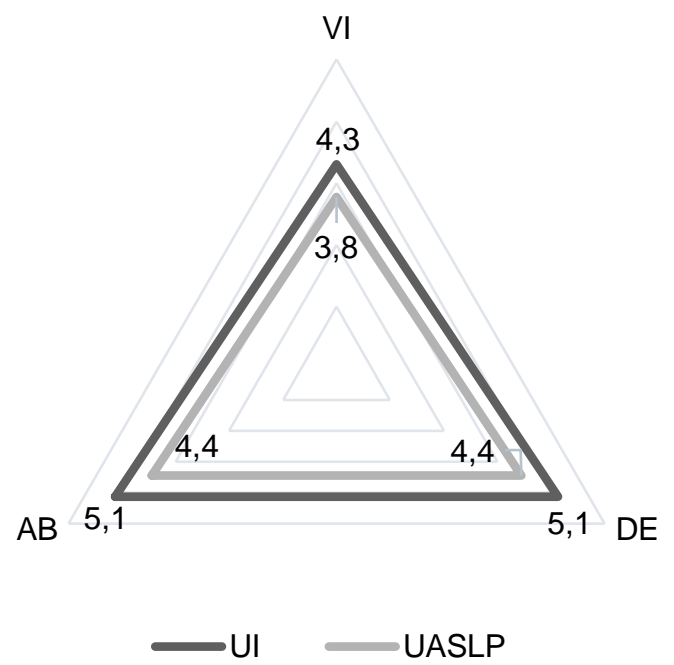

Fig. 1. Comparativo entre Universidad Intercultural y Universidad Autónoma de San Luis Potosí

\section{Análisis de conglomerados de estudiantes universitarios}

Se realizaron análisis para conocer la susceptibilidad de la muestra a la separación por grupos en función del factor dominante $D E$ (f1) o VI y $A B$ (f2). En la tabla 3 se resumen los resultados del análisis de k-medias y se visualizan los perfiles característicos de los conglomerados de universitarios, presentando características opuestas según las puntuaciones a cada factor. Complementariamente, se observa que el nivel de significancia de los factores (95\%) permite detallar una representatividad en sus valores. Adicionalmente, se tomaron en cuenta los valores del estadístico $\mathrm{F}$ que señalan qué factor $\mathrm{DE}$ tiene un mayor peso en la variación de los grupos, así como en los dos perfiles distintivos de cada grupo de estudiantes universitarios, a partir de los puntajes de las características de los centroides de cada grupo y factor.

Tabla 3. Resultados de los centros de conglomerados finales

\begin{tabular}{|l|c|c|c|c|c|r|r|}
\hline \multicolumn{4}{|c|}{ Clúster } & \multicolumn{4}{|c|}{ ANOVA } \\
\hline Segmento & desalentados & animados & \multicolumn{2}{|c|}{ Clúster } & \multicolumn{3}{c|}{ Error } \\
\hline \multicolumn{1}{|c|}{$\%$} & 36.323 & 63.677 & Media cuadrática & gl & Media cuadrática & gl & $\mathrm{F}$ \\
\hline $\mathrm{DE}$ & -0.93177 & 0.5315 & 110.438 & 1.000 & 0.341 & 221.000 & 323.552 \\
\hline VI y AB & -0.44907 & 0.25616 & 25.652 & 1.000 & 0.608 & 221.000 & 42.192 \\
\hline
\end{tabular}


El conglomerado de desalentados considera la menor proporción de la muestra de estudiantes universitarios $(36.32 \%)$ y su mayoría la integran alumnos de la UASLP $(80.2 \%)$ pertenecientes a la carrera de mercadotecnia (48.1\%), administración pública (27.2\%) y turismo sustentable (24.7\%) en su mayoría son mujeres $(72.8 \%)$ de una edad entre los 18 (23.5\%), 20 (16\%) y 17 (14.8\%) años; y se concentran en el segundo semestre de sus carreras (69.1\%). El segmento de estudiantes desalentados presenta menores actitudes referentes a sentirse con energía y felices al momento de realizar las actividades inherentes a su carrera; no encuentran un significado a sentirse entusiasmados con sus estudios. Las puntuaciones negativas también se señalan al no sentirse orgullosos de lo que están estudiando ni tener interés en ir a las clases o sentirse inspirados.

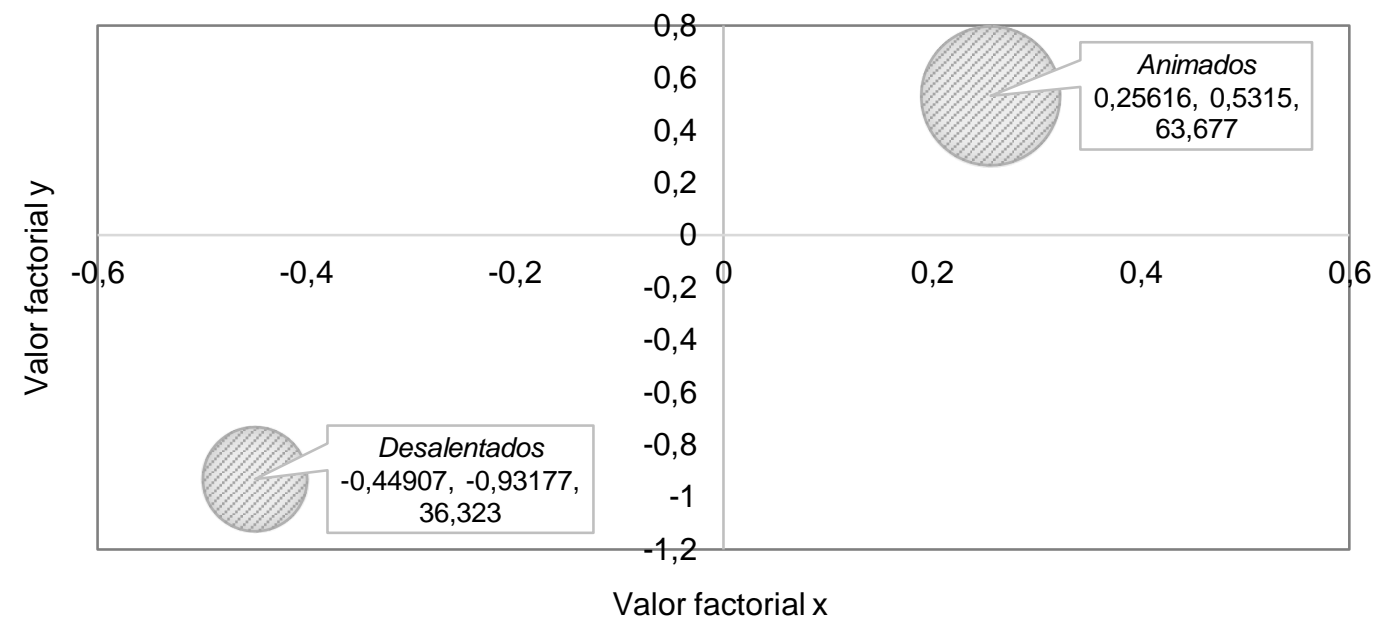

Fig. 2. Representación gráfica de los conglomerados de estudiantes

En contraparte el conglomerado de animados representa la mayor proporción de la muestra $63.67 \%$ y en su mayoría pertenecen a la Universidad Intercultural (54.2\%), cursando la carrera de administración pública (38\%), turismo sustentable (31.7\%) y mercadotecnia (28.9\%). El segmento de estudiantes mayormente lo componen mujeres (72.5\%) entre una edad de $22(25.4 \%), 20(15.5 \%)$ y $18(14.1 \%)$ años y cursan el segundo (39.4\%) y cuarto semestre de sus carreras. El segmento de los animados presenta actitudes positivas, ambos factores especialmente a la $D E$ y $A B$, lo que se interpreta como estudiantes que creen que la Licenciatura que estudian tiene un alto significado y se sienten muy entusiasmados, así como inspirados a realizar cosas nuevas a partir de sus estudios, por ello, están orgullosos de estudiar su carrera y se sienten inmersos en sus estudios. Complementariamente, los animados experimentan fuerza y vigor cuando estudian o se conectan en sus clases, así como felicidad, energía y entusiasmo con las tareas relacionadas con sus estudios (ver figura 2).

\section{Análisis inferencial de las variables}

En complemento y para encontrar un mayor entendimiento al CA, esta sección tiene como objetivo identificar las posibles relaciones estadísticamente significativas entre indicadores de las dimensiones de la variable CA, así como entre estas y las variables sociodemográficas. En este sentido, se realizaron las siguientes pruebas de hipótesis (ver tabla 4). H1: Los alumnos que están orgullosos de su carrera se sienten felices cuando realizan sus tareas. Existe una relación directa (0.69) entre la variable orgullo y felicidad relacionada con las tareas que se desarrollan en la carrera que se cursa $(p<0.01)$. La hipótesis se acepta; H2: Estar entusiasmado con la carrera influye en sentirse feliz al momento de realizar tareas. Se identifica una relación directa (0.65) entre la variable entusiasmo y felicidad en las tareas que se realizan $(p<0.01)$. La hipótesis se acepta; H3: Sentirse orgulloso de la carrera conlleva a contar con fuerza y vigor. Se verifica una relación directa (0.59) $(\mathrm{p}<0.01)$. La hipótesis se acepta; y H4: Cuando el estudiante se abstrae en sus estudios se siente fuerte y vigoroso. Existe una relación directa (0.56) entre la dimensión vigor y abstracción $(p<0.01)$, debido a que los alumnos que se encuentran estudiando normalmente se olvidan de su alrededor para concentrarse en las tareas que están realizando, esta condición puede favorecer el contexto del aprendizaje. Se acepta la hipótesis.

Adicionalmente, se pudo determinar en la población que pertenecer a la UI se asocia con mayores niveles de CA en sus tres dimensiones con puntuaciones en promedio 15\% mayores con respecto a la UASLP. También, que la variable semestre condiciona los niveles de CA en sus tres dimensiones; a medida que se avanza en la carrera aumentan los niveles de CA, lo cual coincide con lo reportado por Loscalzo y Giannini (2019) en estudiantes italianos. Se tuvieron resultados similares a los Serrano, et al. (2019) en estudiantes españoles, en el sentido de que la edad y sexo no impactan en los niveles de CA. 
Las formas de comunicación, las plataformas digitales de aprendizaje y el dominio de competencias digitales han sido estudiadas recientemente (Katz, et al., 2021) como mecanismos de reconocimiento a la medida de transición forzada a la enseñanza digital o en línea, no obstante, la salud mental de alumnos, especialmente el compromiso académico ha requerido una minuciosa observación.

Tabla 4. Relaciones estadísticamente significativas

\begin{tabular}{|l|l|l|c|c|}
\hline Variable 1 & Variable 2 & Prueba & Valor & $p$ \\
\hline DE4 & AB3 & C de Pearson & 0.693 & 0.000 \\
\hline AB3 & DE2 & C de Pearson & 0.654 & 0.000 \\
\hline VI2 & DE4 & C de Pearson & 0.598 & 0.000 \\
\hline VI2 & AB2 & C de Pearson & 0.567 & 0.000 \\
\hline VI (valor medio) & Institución & U de Mann-Whitney & & 0.000 \\
\hline DE (valor medio) & Institución & U de Mann-Whitney & & 0.000 \\
\hline AB (valor medio) & Institución & U de Mann-Whitney & & 0.000 \\
\hline VI (valor medio) & Semestre & H de Kruskal-Wallis & & 0.032 \\
\hline DE (valor medio) & Semestre & H de Kruskal-Wallis & & 0.004 \\
\hline VI (valor medio) & Semestre & H de Kruskal-Wallis & & 0.004 \\
\hline VI (valor medio) & Clúster & U de Mann-Whitney & & 0.000 \\
\hline DE (valor medio) & Clúster & U de Mann-Whitney & & 0.000 \\
\hline VI (valor medio) & Clúster & U de Mann-Whitney & & 0.000 \\
\hline
\end{tabular}

\section{DISCUSIÓN}

Los resultados del presente permiten explicar el CA de estudiantes universitarios mexicanos de acuerdo con sus puntuaciones en $V I, D E$ y $A B$ así como presentar las siguientes conclusiones: 1) se encontraron diferencias estadísticamente significativas entre pertenecer a la UI con mayores niveles y la UASLP con puntuaciones inferiores; 2) el uso del UWES-S en su versión en español para el contexto potosino mexicano fue validado mediante un modelo bifactorial que cumplió con la validez convergente y discriminante, lo cual coincide con los resultados de Meng y Jin (2017) y Portalanza, et al. (2017) y 3) estructuralmente, el modelo bifactorial conjunta los tres elementos del CA.

La $A B$ se presenta como un ítem complementario para el factor 2 lo que plantea que los estudiantes han experimentado la $A B$ al sentirse felices -o no- cuando están realizando tareas concernientes con sus estudios (AB3), sin embargo, no se encontró suficiente varianza entre los ítems de $A B$ que permitiera identificar un factor dentro del modelo propuesto por Schaufeli y Bakker (2004) lo que permite reconocer que el factor $A B$ no está siendo experimentado por los estudiantes y que el único ítem de $A B$ que se encuentra en el modelo bifactorial $(A B 3)$ tiene una relación entre el orgullo que sienten al pertenecer a la carrera en la que estudian (DE4). Los resultados evidencian que en el contexto mexicano sobresale la dimensión VI, lo que implicaría energía y resistencia mental de los estudiantes mientras se realizan actividades inherentes a su carrera, así como un deseo por esforzarse incluso ante obstáculos.

La tipología de estudiantes animados presenta la mayor cantidad de estudiantes que tienen puntuaciones altas en el factor de DE que, profundizando en el concepto de Portalanza, et al. (2017), sugiere que los universitarios están altamente implicados en el estudio y se sienten orgullosos y entusiasmados por las asignaturas y actividades que se encomiendan. En contraparte conviven alumnos universitarios con actitudes de desagrado y desmotivación por la carrera que están estudiando, lo cual refleja la poca disposición del estudiante en ser reflexivo, tener poca o nula apertura en realizar esfuerzos que le permitan comprender, así como no tener una manifestación de inspiración ni orgullo o encontrar un significado en su carrera de licenciatura. Esta situación la denominan Lara, et al. (2018) como desafección escolar (disengagement), en la cual la DE es el factor que requiere atención. Ambas tipologías conviven en el mismo ambiente escolar y permiten identificar a los estudiantes que puedan sentirse desmotivados, con propensión en deserción y/o abandono escolar.

La dimensión $A B$ incide en el $\mathrm{VI}$; cuando el estudiante experimenta $A B$ al desempeñar sus actividades académicas mientras que dedicación permite a los alumnos un mayor involucramiento dentro de sus actividades académicas es probable que se conformen en el grupo de alentados lo cual evidencia la 
importancia del seguimiento a través de los procesos de selección e ingreso a las universidades para identificar los niveles del CA por la carrera que va a optar, dado que esto influirá en su trayectoria académica. Aparentemente, en la selección de estudiantes del presente estudio no se encontró suficiente varianza que demostrara actitudes de $A B$ en sus estudios, sin embargo, sí se encontró que al sentirse orgullosos con su carrera es probable se sientan felices realizando actividades inherentes a su profesión, en otras palabras, los estudiantes demostraron vigor y dedicación en mayor medida, pero no conducen necesariamente a un estado de $A B$ en términos de $C A$.

El presente estudio resalta la importancia de la medición y el reconocimiento del compromiso académico en donde hay evidencia para afirmar que existe una dicotomía entre los niveles de CA que experimentan alumnos universitarios de diferentes instituciones, en este sentido Da Rocha, et al. (2016) consideran necesario conocer y reconocer el perfil de los estudiantes para así definir acciones que atiendan aspectos actitudinales, cognitivos y emocionales involucrados en estimular el compromiso académico, sobre todo en ambientes en línea. Los resultados del presente estudio permiten asumir la importancia de procurar que permanezca el CA en los estudiantes durante su trayectoria académica para que así consoliden su desarrollo integral. Considerando el contexto en donde se desenvuelve el estudio -modalidad de enseñanza-aprendizaje digital forzada debido a la contingencia sanitaria- de acuerdo con Rodríguez y Oramas (2017) es imprescindible la función de los docentes y de las instituciones para facilitar y mejorar el clima emocional del aula con el propósito de que los alumnos desarrollen efectivas competencias profesionales inherentes a sus carreras.

\section{CONCLUSIONES}

De este estudio, de sus resultados y de la discusión, se puede extraer las siguientes conclusiones principales: (1) el diseño de estrategias pedagógico-didácticas para la educación en línea presentan un desafío en cuanto a los niveles de $C A$ debido a que es evidente que una gran parte de alumnos experimentan una falta de $A B$ por lo que es muy posible que este fenómeno se explique desde que la adaptación de una didáctica digital la cual no era el fin educativo de los programas en donde se encuentran inscritos y por ello exista una falta de organización, capacitación para docentes, alumnos y padres de familia sobre el apoyo de las tecnologías, planificación docente y apoyo pedagógico; (2) debe haber un reconocimiento legítimo de las instituciones sobre el ambiente de transición forzado actual de espacios presenciales a espacios digitales, en especial la adecuación de un plan sistemático que incluya al personal, la institución, los alumnos y los agentes que asumen un papel activo en la gestión de la adaptación en la enseñanza-aprendizaje digital, en especial enfocar los esfuerzos en la capacitación en competencias digitales en su dimensión pedagógica e instrumental. Así como la evaluación de la posibilidad de adaptación desde directrices escolares, de contenidos, infraestructura, acceso a TIC, competencia docente y competencia digital de estudiantes y docentes, de manera que se considere el CA como un mecanismo de diagnóstico en la eficiencia de la adaptación y correcta transición; (3).Un buen inicio contextual para reconocer las condiciones que presentan los alumnos desde el VI, AB y DE para planificar mecanismos para incrementar valores que pueden resultar en un mayor aprovechamiento y experiencia de aprendizaje por parte del alumno en los espacios de formación digitales, por lo que es necesario reconocer las condiciones en las que se encuentra el alumno en materia de CA que conduzcan al docente, las instituciones y el propio alumno a describir escenarios y planificar, diseñar y definir mecanismos de interacción, así como herramientas de enseñanza tomando en cuenta las condiciones de CA encontradas; y (4) a partir de la evidencia también se puede resaltar que el CA no había sido medido en condiciones transicionales como las que se viven actualmente -adaptación forzada de espacios de formación digitales- por lo que no existen estudios que descarten la evidencia encontrada.

\section{REFERENCIAS}

Arias, P.R., García, F.E., y Reivan-Ortiz, G., Propiedades psicométricas de la escala de compromiso académico versión abreviada (UWESS-9) en estudiantes ecuatorianos, Ajayu, 18(1) 1-23 (2020)

Aspeé, J.E., González, J.A., y Cavieres-Fernández, E.A., El compromiso estudiantil en educación superior como agencia compleja, http://dx.doi.org/10.4067/S0718-50062018000400095, Form. Univ., 11(4), 95-108 (2018)

Borzone, M.A., Autoeficacia y vivencias académicas en estudiantes universitarios, https://doi.org/10.14718/ACP.2017.20.1.13, Act. Colom. Psicol., 20(1), 266-274 (2017)

Cachón-Zagalaz, J., Lara-Sánchez, A., y otros tres autores, Propiedades psicométricas de la Utrecht Work Engagement Scale en estudiantes de educación, http://dx.doi.org/10.14349/sumapsi.2018.v25.n2.3, Suma Psicol. 25, 113-121 (2018)

Carmona-Halty, M.A., Schaufeli, W.B., y Salanova, M., The Utrecht work engagement scale for students (UWES-9S): factorial validity, reliability, and measurement invariance in a Chilean sample of undergraduate university students, https://doi.org/10.3389/fpsyg.2019.0101, Front. Physiol.,10, 1-5 (2019)

Cotán-Fernández, A., Martínez-Valderrey, V., y otros tres autores, El trabajo colaborativo online como herramienta didáctica en Espacios de Enseñanza Superior (EEES). Percepciones de los estudiantes de los Grados en Educación Infantil y Primaria, https://doi.org/10.1344/RIDU2020.12.9, RIDU, 12, 82-94 (2020) 
Da Rocha, L., Gomes, A. S., y de Melo, I. J., Effectiveness of gamification in the engagement of students, https://doi.org/10.1016/j.chb.2015.11.021, Comput. Hum. Behav., 58, 48-63 (2016).

Díaz, A., Lescano, G.S., y Paz, W.R., Relación entre valores universales y compromiso académico en cadetes del Ejército y la Marina de Perú, https://doi.org/10.21830/19006586.609, Rev. Cient. Gen. José María Córdova, 18(31) 495$521(2020)$

Domínguez-Lara, S., Sánchez-Villena, A.R., y Fernández-Arata, M., Psychometric properties of the UWES-9S in Peruvian college students, http://www.doi.org/10.14718/ACP.2020.23.2.2, Act. Colom. Psicol., 23(2), 7-23. (2020)

Dumford, A.D., y Miller, A.L., Online learning in higher education: exploring advantages and disadvantages for engagement, https://doi.org/10.1007/s12528-018-9179-z, J. Comput. High. Educ., 30, $452-465$ (2018)

Hinrichs, C.P., Ortiz, L.E., y Pérez, C.E., Relación entre el bienestar académico de estudiantes de kinesiología de una universidad tradicional de Chile y su percepción del ambiente educacional, http://dx.doi.org/10.4067/S071850062016000100012, Form. Univ., 9(1), 109-116 (2016)

Hooper, D., Coughlan, J., y Mullen, M. R., Structural equation modelling: guidelines for determining model Fit, https://doi.org/10.21427/D7CF7R, Electron. J. Bus. Res., 6 (1), 53-60. (2008)

Kahn, W. A., Psychological conditions of personal engagement and disengagement at work, https://doi.org/10.5465/256287, Acad. Manage. J., 33(4), 692-724 (2008)

Katz, V. S., Jordan, A. B., y Ognyanova, K., Digital inequality, faculty communication, and remote learning experiences during the COVID-19 pandemic: a survey of U.S. undergraduates, https://doi.org/10.1371/journal.pone.0246641, PLoS ONE, 16(2), 1-16 (2021)

Kim, W.H., Park, J.G., y Kwon, B., Work engagement in South Korea: validation of the Korean version 9-Item Utrecht work engagement scale, https://doi.org/10.1177/0033294117697085, Psychol. Rep., 120(3), 561-578 (2017)

Lara, L., Saracostti, M., y otros cinco autores, Compromiso escolar: desarrollo y validación de un instrumento, Rev. Mex. de Psicol., 35(1), 52-62 (2018)

Loscalzo, Y., y Giannini, M., Study engagement in Italian university students: a confirmatory factor analysis of the UWES-Student version, https://doi.org/10.1007/s11205-018-1943-y, Soc. Indic. Res., 142, 845-854 (2019)

Martin, F., y Bolliger, D.U., Engagement matters: student perceptions on the importance of engagement strategies in the online learning environment, http://dx.doi.org/10.24059/olj.v22i1.1092, Online Learn., 22(1), 205- 222 (2018)

Mendes da Silva, J.O., Pereira, G.A., y otros dos autores, Engajamento entre estudantes do ensino superior nas ciências da Saúde, https://doi.org/10.1590/1981-52712015v42n2RB20170112, Rev. Bras. Edu. Med., 42(2),15-25 (2018)

Meng, L., y Jin, Y. A., Confirmatory factor analysis of the Utrecht work engagement scale for students in a Chinese sample, https://doi.org/10.1016/j.nedt.2016.11.017, Nurse Educ. Today, 49, 129- 134 (2017)

Parra, M., Lay, N., y otros dos autores, Factores que intervienen en el nivel de ausentismo del personal que labora en un centro de llamado de Barranquilla (Colombia), http://dx.doi.org/10.4067/S0718-07642020000600077, Inf. Tecnol., 31(6), 77-86 (2020)

Parra, P. P., Relación entre el nivel de engagement y el rendimiento académico teórico/práctico, Revista Educación, Ciencia y Salud, 7(1), 57-63 (2010)

Portalanza, C.A., Grueso, M.P., y Duque, E.J., Propiedades de la Utrecht work engagement scale (UWES-S 9): análisis exploratorio con estudiantes en Ecuador, https://doi.org/10.15446/innovar.v27n64.62374, Innovar-Rev. Cienc. Ad., 27(64), 145-155 (2017)

Rodríguez, G. L., y Oramas, V. A., Evaluación del compromiso estudiantil en un grupo de ciencias de la actividad física y salud, RECUS, 2(1), 47-50 (2017)

Rodríguez-Montalbán, R., Martínez-Lugo, M., Sánchez-Cardona, I., Análisis de las propiedades psicométricas de la Utrecht work engagement scale en una muestra de trabajadores en Puerto Rico, https://doi.org/10.11144/Javeriana.UPSY13-4.appu, Univ. Psychol., 13(4), 1255-1266 (2014)

Schaufeli, W. B., y Bakker, A. B., Utrecht work engagement scale, preliminary manual (1.1), Utrecht: Occupational Health Psychology Unit, Utrecht University (2004)

Schaufeli, W. B., Salanova, M., y otros dos autores, The measurement of engagement and burnout: a two sample confirmatory factor analytic approach, https://doi.org/10.1023/A:1015630930326, J. Happiness Stud., 3(1), 71-92 (2002)

Serrano, C., Andreu, Y., y otros dos autores, Psychometric properties of Spanish version student Utrecht work engagement scale (UWES-S-9) in high-school students, https://doi.org/10.1017/sjp.2019.25, Span. J. Psychol., 22(21) 1-9 (2019)

Tayama, J., Schaufeli, W., y otros dos autores, Validation of a Japanese version of the work engagement scale for students, https://doi.org/10.1111/jpr.12229, Jpn. Psychol. Res., 61(4) 262-272 (2019)

Wickramasinghe, N.D., Dissanayake, D.S., y Abeywardena, G.S., Validity and reliability of the Utrecht work engagement scale-student version in Sri Lanka, https://doi.org/10.1186/s13104-018-3388-4, Res. Notes, 11(277) 1-6 (2018) 